\title{
Lectura de las proporciones utilizadas por el maestro Ayora en las iglesias salón valencianas del XvIII
}

\section{Reading of the proportions used by master architect Ayora in the 18th century Valencian churches}

\author{
B. Sáez Riquelme $^{(*)}$, Á. M. Pitarch Roig ${ }^{(* *)}$
}

\section{RESUMEN}

En el presente artículo se analiza la obra del Maestro Arquitecto Joseph Ayora y su saga, y su aportación a los templos valencianos del xviII, concebidos siguiendo el modelo hallenkirchen. Nacido en un pequeño pueblo de Teruel pronto dejará indicios de su actividad como tracista y constructor en la provincia de Castellón, donde al menos se le relaciona con once templos, de los cuales cinco son columnarios. En las Iglesias de Castell de Cabres y Cinctorres ha sido documentada la autoría de sus trazas, en Vila-real su aportación fue parcial, mientras que en las de Benifairó de les Valls y Suera se le presupone.

El análisis gráfico basado en el exhaustivo levantamiento de planos de la obra patrimonial construida y en el estudio comparado de sus trazados reguladores, su métrica y su composición, se muestra como una herramienta veraz, capaz de esclarecer presuntas autorías y desvelar el patrón de diseño de los tracistas.

Palabras clave: proporción, trazados reguladores, metrología, hallenkirchen, patrimonio.

\section{ABSTRACT}

In the present article it is analyses the building work of the family's Master Architect Joseph Ayora, and his contribution to the Valencian temples of the XVIIIth conceived following the hallenkirchen model. This master mason was born in a little village of Teruel, he will leave early indications of his activity as tracer and builder in Castellón's province, where at least he is related to eleven temples, of which five are hall churches. In the Churches of Castell de Cabres and Cinctorres has been documented the authorship of its traces, in Vila-real its contribution was partial, whereas in the churches of Benifairó de les Valls and Suera it is presupposed.

The graphical analysis based on the exhaustive raising of planes of the patrimonial constructed work and on the compared study of its regulatory tracings, its metrics and its composition, appears as a veracious tool, capable of clarifying supposed authorships and reveals the template of design of the tracers.

Keywords: proportion, regulating lines, metrology, hallenkirchen, heritage.

${ }^{(*)}$ Departamento de Ingeniería de Sistemas Industriales y Diseño. Universitat Jaume I (España).

(**) Departamento de Ingeniería Mecánica y Construcción. Universitat Jaume I (España).

Persona de contacto/Corresponding author: bsaez@uji.es (B. Sáez Riquelme).

ORCID: https://orcid.org/o000-0003-4502-8106 (B. Sáez Riquelme); https://orcid.org/oooo-0002-1091-8283

(Á.M. Pitarch Roig)

Cómo citar este artículo/Citation: Sáez Riquelme, B.; Pitarch Roig, A.M. (2020). Lectura de las proporciones utilizadas por el maestro Ayora en las iglesias salón valencianas del xviI. Informes de la Construcción, 72(557): e330. https://doi.org/10.3989/ic.62812

Copyright: ( ) 2020 CSIC. Este es un artículo de acceso abierto distribuido bajo los términos de la licencia de uso y distribución Creative Commons Reconocimiento 4.o Internacional (CC BY 4.0). 


\section{INTRODUCCIÓN}

Las iglesias de Planta salón, Hallenkirchen o Iglesias Columnarias, constituyen un tipo de arquitectura religiosa extendido por parte de Europa $(1,2)$ y América $(3,4)$.

Su expansión en el Reino de Valencia, mayoritariamente en tierras castellonenses, se produce principalmente en el siglo xviII,conlaedificación dediecisietetemplosquedandofrenadaa finales de la misma centuria, por la influencia de las Reales Academias en el trazado y dirección de las obras arquitectónicas $(4,5)$.

Formalmente se definen por mantener la misma cota de arranque de las bóvedas de las naves, y la conformación de un espacio interior unitario (6).

\subsection{Objetivos}

En el presente artículo se analizan las similitudes y diferencias geométricas y formales de los diversos templos cuya autoría se atribuye al maestro Ayora y su saga, con objeto de ratificar o no la posible autoría de estos.

\subsection{El maestro Ayora y su aportación a las Iglesias Salón Valencianas del XVIII}

Los Ayora formaban un clan familiar compuesto de tracistas y constructores. Son los autores o han intervenido en varios de los templos de planta salón erigidos durante la segunda mitaddelacenturiadelxvirien el ReinodeValencia, perdurando la duda de su aportación en otros.

Cabe destacar la figura de Joseph Cristóbal Ayora, nacido en Las Parras de Castellote entre 1710 y 1725. "Maestro Arquitecto" por el gremio albañiles y pintores de Morella en 1755 (7), a quien se le atribuyen diversas obras en poblaciones del norte de la provincia de Castellón, y en la comarca de la Plana Baja (7, pp. 50, 56, 202, 206, 207).

Su hermano Juan Antonio Ayora, es citado como "Maestro Director de Arquitectura" hecho que pudiera hacer pensar en que pasó por la Academia (7, p. 46). Desarrolla su vida profesional inicialmente en la comarca castellonense de Els Ports, y posteriormente en diferentes poblaciones aragonesas.

\subsection{Metodología de análisis}

Para acometer este estudio resulta necesario realizar el levantamiento de planos de todas las construcciones a realizar. Las técnicas de este y la documentación gráfica deben ser acordes con la finalidad y objetivos de la representación que se deseen alcanzar (8). En este sentido se ha optado por la realización de plantas y secciones, basadas en la combinación de técnicas (9). En concreto para los levantamientos gráficos se ha combinado la utilización de una estación total no reflectante, Trimble 3600 Total Station, con una presión de $1 \mathrm{~mm}+$ $1 \mathrm{ppm}$, con la que se han tomado las medidas generales, con aplicaciones fotográmetricas y mediciones manuales para los elementos de detalle. Ello ha permitido obtener una precisión en las dimensiones generales del levantamiento del orden de $0,1 \%$ y para las de detalle del orden del $0,5 \%$, salvo que en algún caso pudieran producirse errores accidentales, pero se entiende que estos deberían ser puntuales y se han sido de- tectados y corregidos durante el dibujo y análisis geométrico realizado.

Los levantamientos realizados permiten determinar la dispersión de las dimensiones, entendida como el procentaje que representa la máxima diferencia respecto de la media, de todos los elementos que deberían tener la misma dimensión, medidos a ejes.

La geometría final permite plantear diversas hipótesis de modulación y trazado susceptibles de haber sido utilizados en la definición de espacios y elementos constructivos (10). Estas se contrastan con la documentación histórica existente, y se comparan entre ellas con objeto de determinar las posibles relaciones que puedan resultar de la autoría de los templos. Así mismo, en algunos casos se analiza el error que se produce entre las dimensiones teórias y el valor realmente medido, expresado como porcentaje.

\subsection{Módulos y trazados reguladores en la arquitectura del siglo $\mathrm{XVIII}$}

Múltiples autores, $(11,12,13,14)$, han aportado contribuciones respecto del análisis geométrico y modulación de las arquitecturas históricas, aludiendo a la necesidad de adoptar alguna referencia para iniciar el trazado y construcción de una iglesia. Generalmente se plantea la disyuntiva, entre recurrir a proporciones dinámicas basadas en trazados geométricos que proporcionan la forma o utilizar proporciones estáticas basadas en un módulo o unidad de medida que permitan dimensionar cada uno de los elementos a partir de un patrón conocido.

La belleza basada en el número ha sido una constante en la arquitectura medieval (11), sin embargo, más allá de elementos puntuales, es poco conocida la incidencia práctica de la geometría en las obras arquitectónicas. Por el contrario, sí lo es, la aplicación de módulos en el dibujo de la planta, siendo éste posiblemente el factor que mejor defina la autoría de cada uno de los maestros.

Respecto de la utilización de trazados reguladores, desde la antigüedad son conocidas y utilizadas diversas proporciones como la proporción Dupla, Ad Quadratum o $\sqrt{ } 2$ y la proporción Aurea, o combinaciones de ellas, que forman parte de la arquitectura culta $(15,16)$.

En la difusión de las proporciones y trazados geométricos a través de los tratados de arquitectura clásica destaca el hecho de que los griegos ya conocían las nociones geométricas necesarias para la aplicación de proporciones (17), no obstante, su propagación comienza con la publicación de los libros primero y segundo de Serlio sobre Geometría y Perspectiva, en París a mediados del siglo xvi (18) quien define diversas

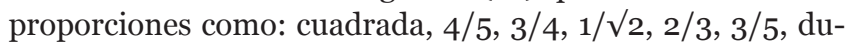
pla. Aunque no fue traducido al castellano el primero no tuvo problemas de difusión en España, a lo que se suma la posterior publicación de Juan de Arfe inspirada en él (19), quien también hace referencia a dichas proporciones e incluye las proporciones multiplex, pero en general cabe entenderlas como conceptos abstractos que relacionan proporciones numéricas.

Si bien no existe constancia que los Ayora tuvieran en su poder dichas publicaciones, si parece probable que fueran cono- 
cedores de ellas, puesto que ya formaban parte de la cultura de los arquitectos de la época, de hecho, incluso en los siglos precedentes ya se había producido la difusión de las mismas en Hispanoamérica (20). De otro modo, la relación con otros maestros de obra, como Juan Joseph Nadal en Vila Real, o Bartolomé Ribelles, académicos o Fray Alberto Pina hacen pensar en un constante intercambio de conocimientos.

De ello se deduce la utilización de una serie de proporciones armónicas o estáticas basadas en la relación de números enteros, tales como $1 / 2$ (dupla), 2/3, 3/4, 3/5, 4/5 y unas proporciones irracionales o dinámicas, basadas en construcciones geométricas tales como $1 / \sqrt{ } 2,1 / \sqrt{ } 3,1 / \sqrt{ } 5$ o la proporción áurea $(1+\sqrt{ } 5) / 2$, cuya denominación fue dada en 1835 por el matemático alemán Martín Ohm, lo que lleva a pensar que si bien era una construcción conocida, no se le dio la importancia actual hasta comienzos del siglo xx.

Por otro lado, hasta finales del siglo xviı no se define el metro como unidad de medida, produciéndose su aplicación generalizada a mediados del siglo xIx, por lo que todas las construcciones anteriores se regían por medidas antropomórficas.

Con la desmembración del imperio romano, se pierde la homogeneidad de estas medidas y aparecen variantes regionales (12), no reguladas hasta el siglo xIII, cuando Jaime I instaura un sistema de medidas para todo el reino de Valencia con el Almotacén como responsable de su cumplimiento (21). Este sistema de medida utilizaba el palmo como unidad, y contemplaba la existencia de diversos múltiplos.

En Los Fueros del Reino de Valencia (1547) se establecen las equivalencias entre la cuerda y las brazas (22), y posteriormente, la Real Orden de 1852 (23) determina la correspondencia de medidas por provincias, apreciándose importantes diferencias. En este sentido cabe reseñar que la división provincial se produce a comienzos del siglo xIx, por lo que puede deducirse que la utilización de las unidades de medida no se correspondian exactamente con las divisiones provinciales.

En la tabla 1, se recogen las equivalencias entre la vara o alma y los palmos valencianos (12), así como una relación de medidas utilizadas a lo largo de la historia (24).

Tabla 1. Medidas antropomórficas de referecia.

\begin{tabular}{|l|c|c|}
\hline \multicolumn{3}{|c|}{$\begin{array}{c}\text { MEDIDAS ANTIGUO REINO DE VALENCIA } \\
\text { (López y García 2012) }\end{array}$} \\
\hline \multicolumn{1}{|c|}{ Medidas } & Equivalencias & Metros \\
\hline Vara o alma & $\begin{array}{l}\text { 4 palmos } \\
\text { 3 pies }\end{array}$ & 0,91 \\
\hline Braza & 9 palmos & 2,07 \\
\hline Cuerda & $\begin{array}{l}\text { 45 varas o } \\
\text { 20 brazas }\end{array}$ & 41,40 \\
\hline $\begin{array}{l}\text { Bastón, junco o caña de } \\
\text { Almotacén }\end{array}$ & 6 palmos & 1,36 \\
\hline
\end{tabular}

\begin{tabular}{|l|c|c|}
\hline \multicolumn{3}{|c|}{ MEDIDAS R.O. 9 DE DICIEMBRE 1852} \\
\hline \multicolumn{1}{|c|}{ Localización } & Vara (cm) & Palmo (cm) \\
\hline Teruel & 76,8 & 19,02 \\
\hline Zaragoza & 77,2 & 19,31 \\
\hline Tarragona & 78,0 & 19,50 \\
\hline Castilla & 83,6 & 20,89 \\
\hline Castellón & 90,8 & 22,70 \\
\hline Valencia & 90,8 & 22,70 \\
\hline
\end{tabular}

\begin{tabular}{|l|c|c|c|c|}
\hline \multicolumn{5}{|c|}{ MEDIDAS TRADICIONALES (Soler 2008) } \\
\hline Localización & $\begin{array}{c}\text { Cana } \\
\text { (cm) }\end{array}$ & $\begin{array}{c}\text { Vara } \\
\text { (cm) }\end{array}$ & $\begin{array}{c}\text { Palmo } \\
\text { (cm) }\end{array}$ & $\begin{array}{c}\text { Pie } \\
\text { (cm) }\end{array}$ \\
\hline Aragón & & 77,70 & 19,40 & 25,90 \\
\hline Castilla & & 83,58 & 20,89 & 27,86 \\
\hline Valencia & & 91,00 & 23,00 & 30,33 \\
\hline Cataluña & 155,50 & 77,75 & 19,43 & \\
\hline
\end{tabular}

\section{ANÁLISIS DE LOS TRAZADOS GEOMÉTRICOS}

De las diecisiete iglesias salón valencianas del xviII (25) se analiza el trazado de las cuatro atribuidas al maestro Ayora junto con las de Benifairó de les Valls, Quart de les Valls, y Suera cuya autoría aún está por esclarecer y cuya contextualización temporal puede verse en la tabla 2.

Tabla 2. Templos analizados por su supuesta vinculación con el maestro Ayora.

\begin{tabular}{|l|c|}
\hline \multicolumn{1}{|c|}{ Iglesia } & $\begin{array}{c}\text { Periodo de } \\
\text { actuación }\end{array}$ \\
\hline Iglesia de San Lorenzo de Castell de Cabres & $\sim 1750 ?-1755$ \\
\hline Iglesia de Sant Pere de Cinctorres & $1763-1782$ \\
\hline Arciprestal de Sant Jaume de Vila-real & $1763-1779-1798$ \\
\hline Iglesia de la Asunción de Suera & $1765-1779$ \\
\hline Iglesia de San Gil de Benifairó de les Valls & $1773-1790$ \\
\hline $\begin{array}{l}\text { Iglesia de la Asunción de Les Coves de } \\
\text { Vinromà }\end{array}$ & $1774-1793$ \\
\hline Iglesia de San Miguel de Quart de les Valls & $1776-1796$ \\
\hline
\end{tabular}

\subsection{Iglesia de San Lorenzo de Castell de Cabres}

Fue encargada a José Cristóbal Ayora, quien comenzó las obras en 1750, y las abandonó 5 años más tarde (5, p. 224), bajo su asesoramiento las continuaron los hermanos José y Fernando Molinos, finalizándola este último (7, pp. 107-227).

El edificio conforma un rectángulo de medidas exteriores 27,35 x 16,03 m, con una dispersión del o,77\%, que se corresponde con la traslación de la modulación interior. Resultando una proporción aproximada de 1/1,69, próxima a 3/5 (ver Figura 1).

Del análisis métrico interior, se deduce que, si bien existen importantes irregularidades atribuibles a su ejecución con una dispersión de hasta el 2,2\%. Los tramos de la nave principal siguen una proporción de 3/4, respondiendo el conjunto de la planta a una sencilla retícula de módulo $79,4 \mathrm{~cm}$, que en el crucero corresponde a 8x8 módulos, en los tramos de la nave principal a $6 x 8$ y en las naves laterales a $6 \times 6$. En alzado se aprecia dicha modulación de 6x6 hasta el capitel corintio y de $6 \times 8$ hasta la cumbrera.

Este mismo módulo se repite, de forma aproximada, en la sección del fuste de los pilares 80 x $80 \mathrm{~cm}$. Sin embargo, hay que señalar que no se ha encontrado una correspondencia precisa entre este módulo y los definidos en el resto de los elementos constructivos como los muros de carga de mampostería, con una variación dimensional entre 0,65$0,77 \mathrm{~m}$.

Buscada la correlación de este módulo de 79,4 cm, entre las diferentes unidades antropológicas recogidas en la bibliografía la más próxima es la vara de Teruel o,768 m o la de Tarragona $0,780 \mathrm{~m}$. 


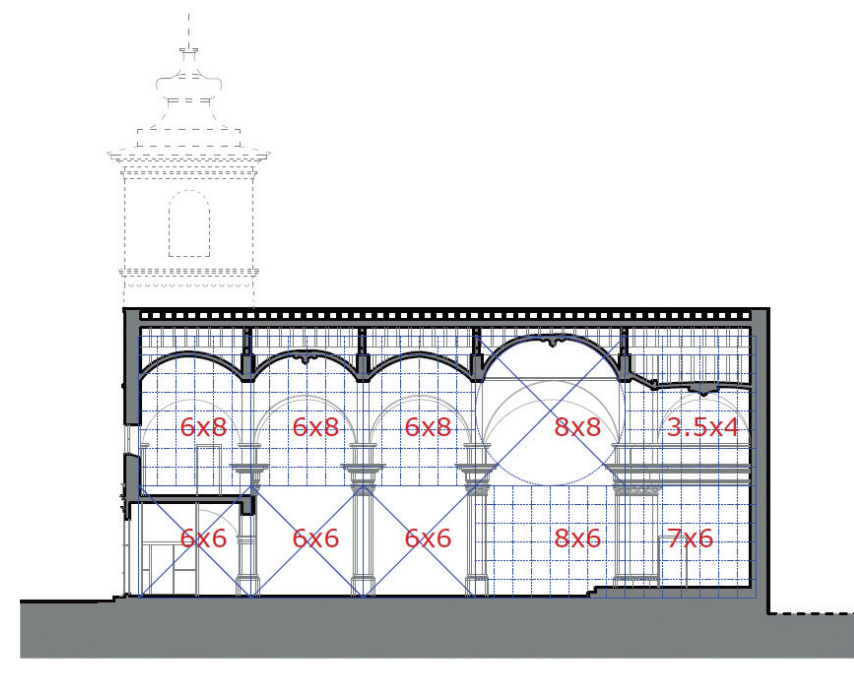

0_- $5 \mathrm{~m}$

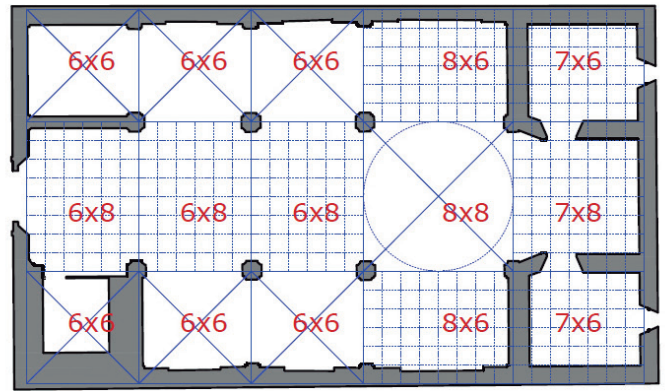

Modulación: 1 vara $=79,4 \mathrm{~cm}$

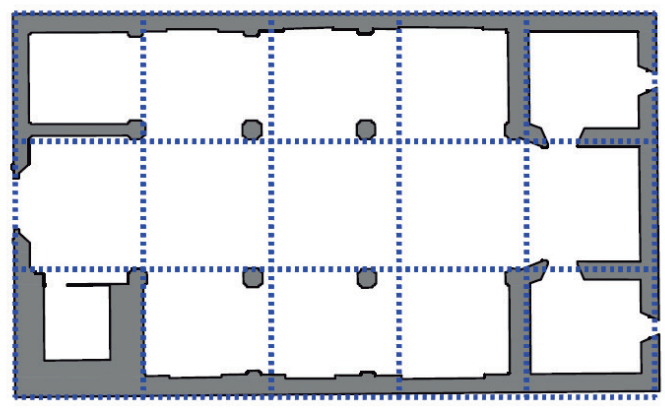

Proporción general del tempo

Figura 1. Modulación Iglesia de Castell de Cabres. Fuente propia.

\subsection{Iglesia de Sant Pere de Cinctorres}

La construcción de este templo se extiende desde 1763 hasta 1782 (5, p. 357), siendo reconstruidas parte de sus bóvedas después de la Guerra Civil española (26).

Las trazas son atribuidas a José Ayora, quien compartiría la dirección de las obras con su tío José Dols y con su hermano, Juan Antonio Ayora, desconociendose la aportación de cada uno de ellos (5, p. 224).

El templo se configura a partir de un rectángulo de dimensiones exteriores $41,10 \times 26,16 \mathrm{~m}$, del que sobresalen 5,62 m, ocupados por la sacristía. Dicho volumen corresponde, aproximadamente, al abatimiendo de las diagonales de un cuadrado, en ambos sentidos $1 /(2 \sqrt{2}-1)$ o $1 / 1,70$. Sin embargo, dicha idealización no encuentra su correspondencia más allá del contorno del templo (ver Figura 2).
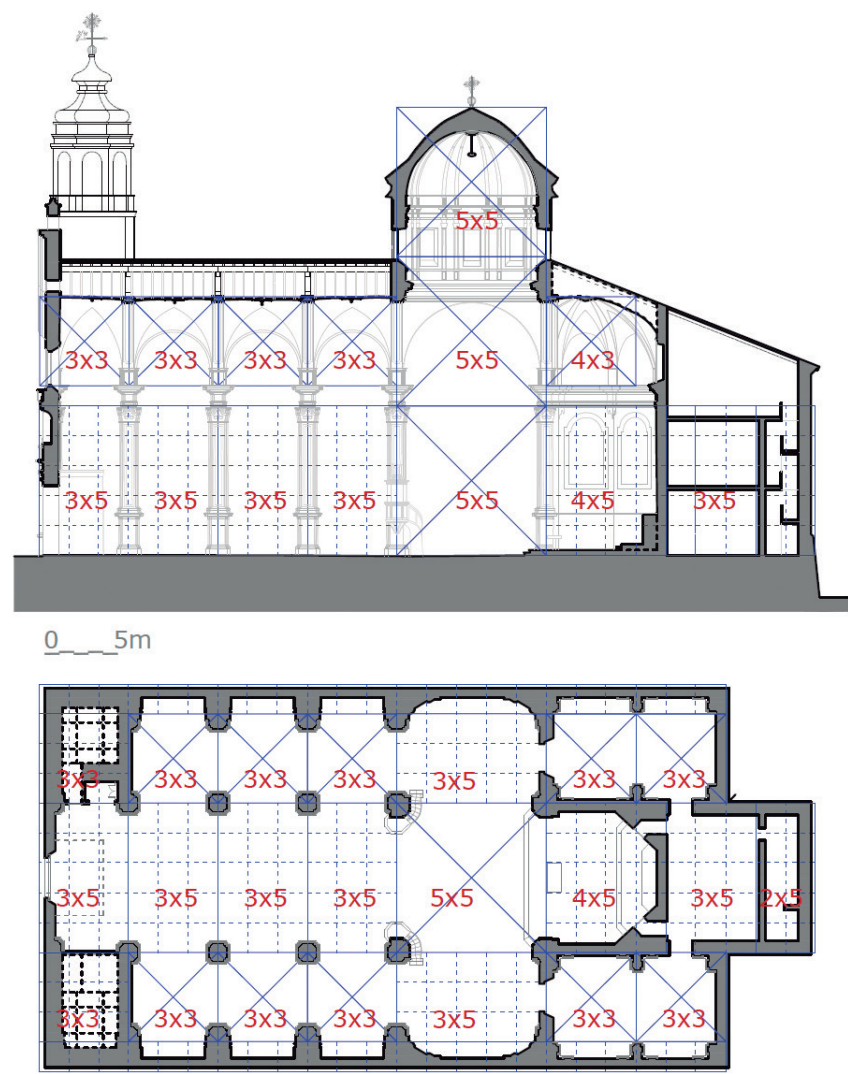

Modulación: 1 braza $=205,4 \mathrm{~cm}=9$ palmos

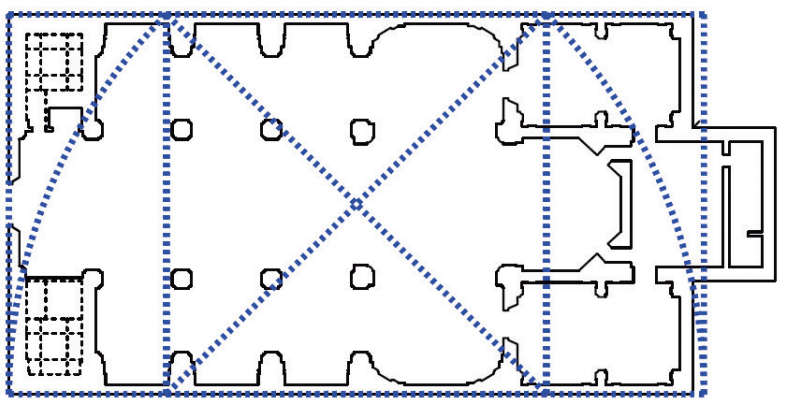

Proporción general del tempo

Figura 2. Modulación Iglesia de Cinctorres. Fuente propia.

Las dimensiones interiores tienen una dispersión insignificante. Del análisis geométrico de la planta se ha podido encontrar una proporción de 3/5 en los tramos de la nave principal y brazos del crucero, que se combinan con un cuadrado de 5 módulos en el crucero, y cuadrados de 3 módulos en los tramos de las naves laterales, siendo el módulo de 2,054 m con errores inferiores al 0,3\%, lo que se aproxima a una braza de 9 palmos de $22,83 \mathrm{~cm}$, próximo al palmo valenciano de $22,76 \mathrm{~cm}$.

La sección longitudinal es susceptible de ser definida, igualmente, por una serie de cuadrados y rectángulos reguladores de 3x5 módulos.

Estas proporciones 3/5 ó 5/8 con la suma del crucero y uno de los tramos responden a la serie de Fibonacci, o a las definidas en los tratados de Serlio y Juan de Arfe.

\subsection{Iglesia arciprestal de Sant Jaume de Vila-real}

Los planos originales (27) están firmados por el arquitecto y académico de mérito por la Academia de San Fernando, Juan 
Joseph Nadal en febrero de 1753. Sin embargo, en 1752 ya se había derribado el templo anterior y colocado la primera piedra (28).

Durante mucho tiempo se mantuvo la creencia de que el tracista había sido Fray José Alberto, «carmelita observante, arquitecto y maestro de obras por Su Majestad» (28, p. 277291), sin embargo, seguramente debieron presentarse varias trazas, siendo finalmente aprobadas las de Nadal (5, p. 346).

Las obras dieron comienzo bajo su dirección, con la colaboraron de sus hijos, hasta 1756, año en que se paraliza la construcción. En 1763, con el templo parcialmente construido, fallece Nadal (7, p. 202). A partir de 1765 asume la ejecución Joseph Dols, durante unos dos años, hasta que su sobrino Joseph Cristóbal Ayora puede hacerse cargo (7, pp. 50, 56). En ellos recae la responsabilidad de cubrir el templo y acabar sus tres portadas.

A partir de 1779 los arquitectos de la Real Academia se hacen cargo de formalizar su conclusión, en primer lugar, Ribelles con la decoración interior, y posteriormente Vicente Gascó revisa los planos para la capilla de la Comunión, siendo el encargado de las obras.

Se trata de una iglesia de grandes dimensiones exteriores de 69,00 x 41,63 m que conforman una planta de proporción aurea, sin embargo, dicha proporción no tiene relación con el trazado interior por lo que puede ser casual (ver Figura 3).

En su interior, se ha medido una dispersión del o,3\% siendo la proporción que rige los tramos de las naves, de 1.4465, que difiere en un $2 \% \sqrt{ } 2$ (Diagón) lo que puede corresponder a un diseño de los planos originales que se ha trasladado a la realidad mediante modulos racionales. En el crucero, los brazos y el presbiterio se puede inscribir un cuadrado, y estos últimos vienen definidos por un circulo inscrito.

En la sección es más complejo encontrar relaciones estáticas que trazados dinámicos. La relación entre la anchura de la nave central y la altura hasta la imposta es de 1/1,27 (29), sin embargo, la altura de las columnas desde el supuesto pavimento original hasta los capiteles compuestos puede definirse mediante la Sección Áurea. Y la altura de las bóvedas interponiendo un ad quadratum. El espacio interior del crucero viene definido por la suma del Diagón y dos cuadrados, encajando el superior con la cúpula.

Aunque en los planos Nadal especifica «palmos del Reyno de Valencia», el análisis del levantamiento realizado permite matizar la utilización de varas valencianas de 90,9 cm entre los ejes, y palmos (22,6 cm) para el ancho de las pilastras. En la capilla de la Comunión se detecta la utilización de varas valencianas para las cotas generales y pies en los tramos de menores dimensiones. En la sección, las alturas de la nave y del crucero vienen definidas por varas valencianas.

Por otro lado, el análisis pormenorizado de la métrica de los diferentes accesos permite establecer que la puerta de la Pescadería, cuyo diseño se adjudica al aragonés Nadal y la supervisión al también aragonés Ayora, se ajusta con bastante precisión a varas aragonesas, mientras que la de la capilla de la Comunión, del valenciano y director de la academia Gascó, a varas valencianas.

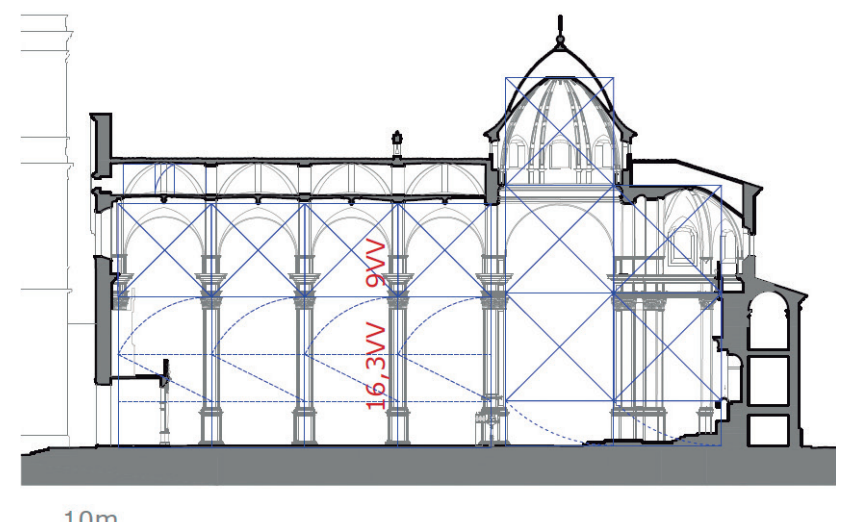

$10 \mathrm{~m}$
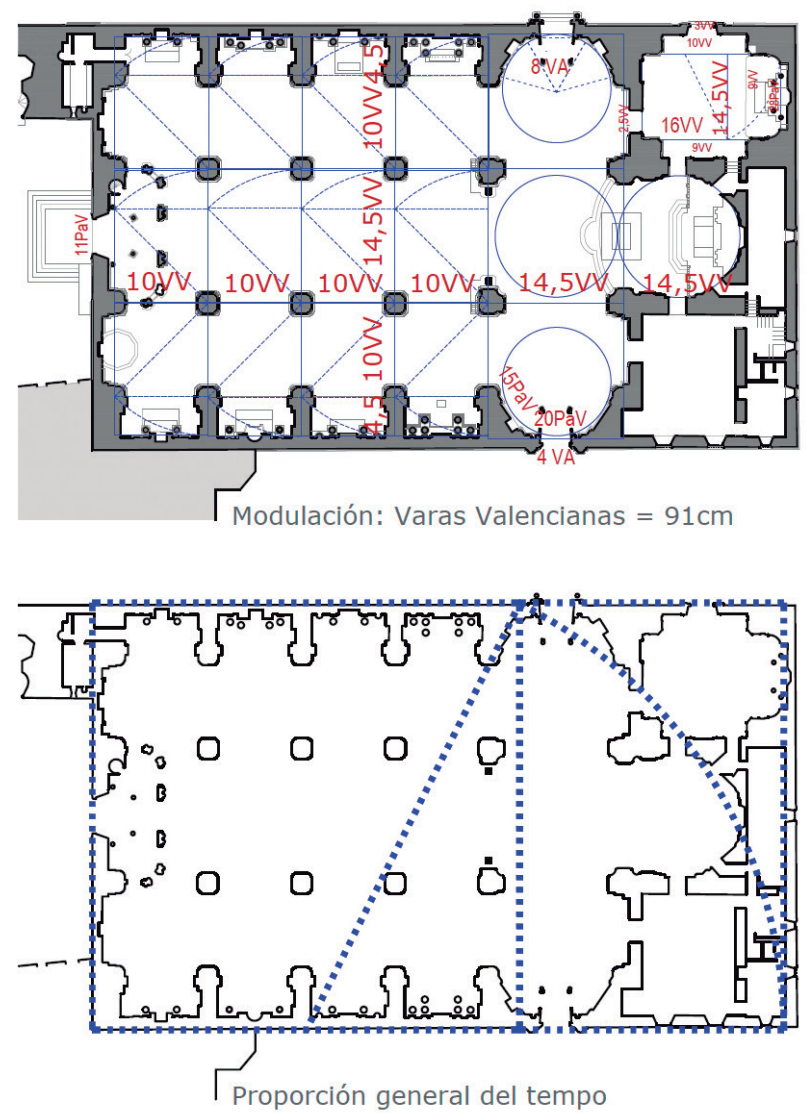

Figura 3. Modulación Iglesia de Vila-real. Levantamiento Navarro y Herráez (30), redibujo propio.

\subsection{Iglesia de San Gil de Benifairó de les Valls}

A pesar de la existencia de discrepancias en las fechas de inicio y finalización, puede considerarse que pertenece a la etapa madura del modelo, aproximadamente, entre 1769 y 1790.

Se desconoce su autoría, sin embargo, se relaciona el inicio de las obras con la fecha en que Joseph Cristóbal Ayora se hace cargo de la arciprestal de Vila-real y el parecido formal entre éstas y la parroquial de Cinctorres, de la que es tracista y constructor (7, p. 96), permiten lanzar la hipótesis que la autoría se deba a éste o a su hijo, vecino de Quart de les Valls, población próxima a Benifairó de les Valls.

El rectángulo definido por las naves tiene unas dimensiones exteriores de 38,33 x 21,18 m, que suprimiendo el tramo de los pies, construido con posterioridad, responde aproximadamente a un rectángulo de proporción áurea (ver Figura 4). 

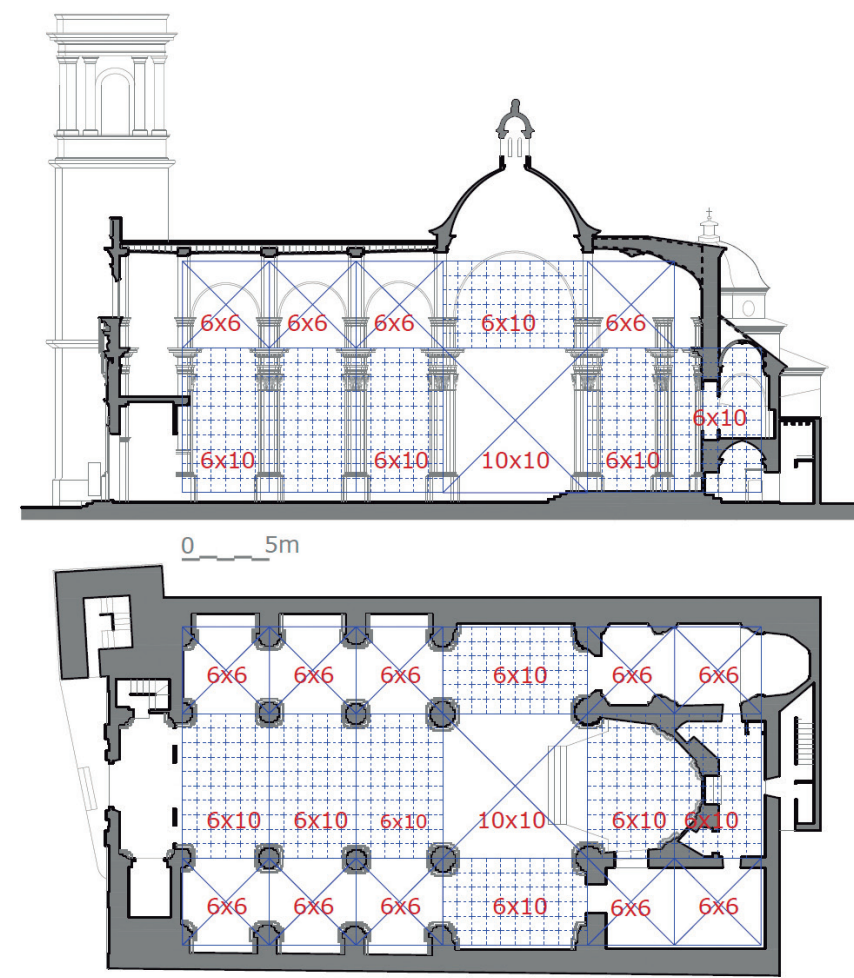

Modulación: 1vara $=83.1 \mathrm{~cm}$

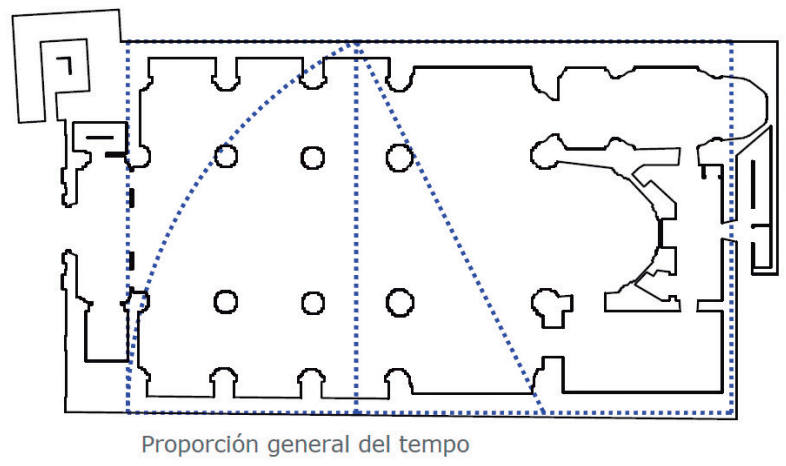

Figura 4. Modulación Iglesia Benifairó de les Valls. Fuente propia.

El análisis métrico desvela una irregularidad en planta, imperceptible a simple vista, pero con una diferencia de $15 \mathrm{~cm}$ entre el ancho de la nave principal en el crucero y los tramos de los pies.

Aun a pesar de ello, en los tramos de la nave principal, se detecta una proporción $3 / 5$, respondiendo el conjunto de la planta a una sencilla retícula al igual que ocurre en Cinctorres. El crucero corresponde a 10x10 módulos, los tramos de la nave principal a 6x10 y las naves laterales a 6x6.

La dispersión máxima en la posición de los pilares respecto de esta modulación es, aproximadamente, del 1,8\%.

La sección encaja, de forma bastante aproximada, con esta modulación, con una altura de 9 módulos hasta el capitel corintio, 11 hasta el arranque de las bóvedas, y 18 hasta el de la cúpula.

Cada uno de los módulos corresponde aproximadamente con $83,1 \mathrm{~cm}$ y por tanto con 4 submultiplos de $20,8 \mathrm{~cm}$.

Se han podido medir diversos espesores de muros, entre 0,95 m y o,61 m, lo que permite confirmar una concepción volumétrica del templo diferente a lo largo del tiempo.
Así mismo, la métrica del tramo de los pies desvela la independencia formal de esta zona, cuya anchura es de 3,09 m, más de $1 \mathrm{~m}$ de diferencia respecto del resto de tramos.

\subsection{Iglesia de la Asunción de Suera}

Se concedió el permiso para su construcción a finales de 1772, y las obras se dilataron hasta 1797 (31), siendo la penúltima en acabarse y coincidiendo en su ejecución con gran parte de las iglesias salón valencianas. La volumetría final la adquiere en 1890, cuando se construyen las salas posteriores al templo.

Las trazas fueron atribuidas inicialmente a fray Alberto Pina, sin embargo, Bautísta (7, pp. 175-176) plantea la posible autoría de Joseph Cristóbal Ayora.

El maestro de obra fue Pau Gonel, al parecer procedente de Fortanete (Teruel), y de una familia con gran tradición en la construcción.

Exteriormente tiene un ancho de 18,94 m y una longitud de $39,62 \mathrm{~m}$ o aproximadamente $26,70 \mathrm{~m}$ si consideramos los tres tramos de los pies, crucero y altar, supuestamente construidos en una primera fase, según la documentación existente así como por las características constructivas.

Los tramos de la nave principal se disponen siguiendo una proporción de $4 / 7$, próxima a $\sqrt{3}$ (ver Figura 5).

El análisis métrico de la planta pone de manifiesto un ligero ensanchamiento de la planta a partir del crucero. Por el contrario, las cotas en altura, casi idénticas, son el resultado de un gran dominio en la ejecución de bóvedas.

Aún con estas diferencias métricas de hasta un 1,0\%, se ha advertido la utilización de una modulación basada en el ancho de los pilares, aproximadamente $1,099 \mathrm{~cm}$, cuyo módulo define los tramos de la nave principal con una modulación de $4 \times 7$.

Dicha modulación puede ser confirmada con la información de las dimensiones originales del templo (31), que permiten deducir que el ancho de los pilares, 5 palmos, se corresponde con el módulo definido en el levantamiento. La única dimensión de la que no se ha podido hallar correspondencia es la longitud general, 132 palmos de largo según la bibliografía, para lo cual tendríamos que excluir el tramo de los pies y el trasagrario, este último añadido en posteriores ampliaciones.

Este análisis permite concluir que el palmo utilizado en este caso mide $21,99 \mathrm{~cm}$, ligeramente diferente de las medidas antropomórficas de referencia, hecho que constata la existencia de variantes en las unidades de medida utilizadas en la época.

\subsection{Iglesia de la Asunción de les Coves de Vinromà}

Se desconoce la fecha de inicio las obras, pero a partir de la documentación existente se deduce que debió ser hacia 1774, y su finalizaron después de 1793. (7, p. 175)

Tampoco queda clara la autoría de las trazas, sin embargo, la dirección de las obras se atribuye a Andrés Moreno, y posteriormente a Fray Joaquín del Niño Jesús, participando de manera puntual Bartolomé Ribelles. Por otro lado, la semejanza del diseño de la portada con las de Cinctorres y San Marcos de 


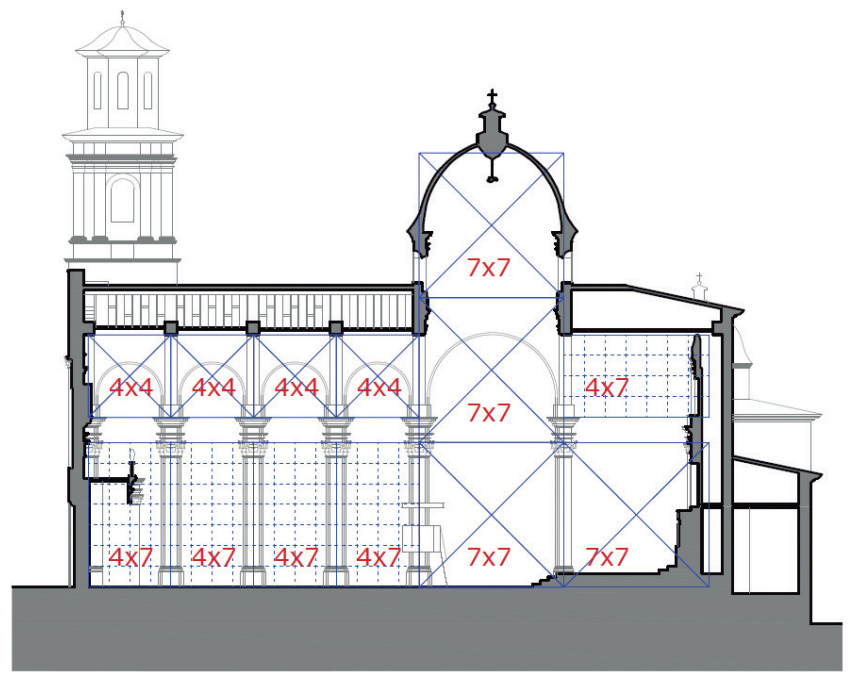

$\underline{0}-\_-{ }^{5 m}$

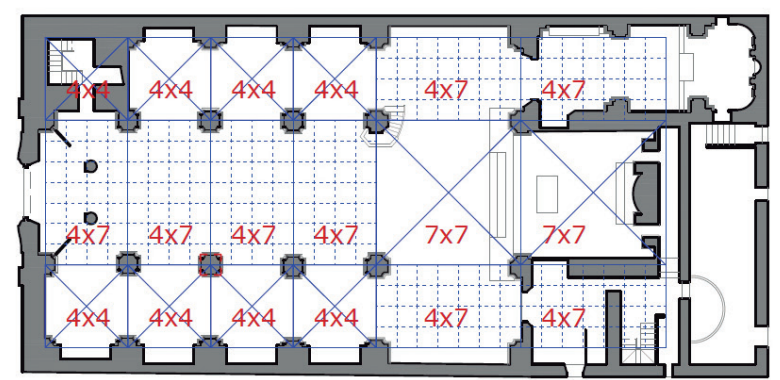

Modulación: 1vara $=109,9 \mathrm{~cm}$

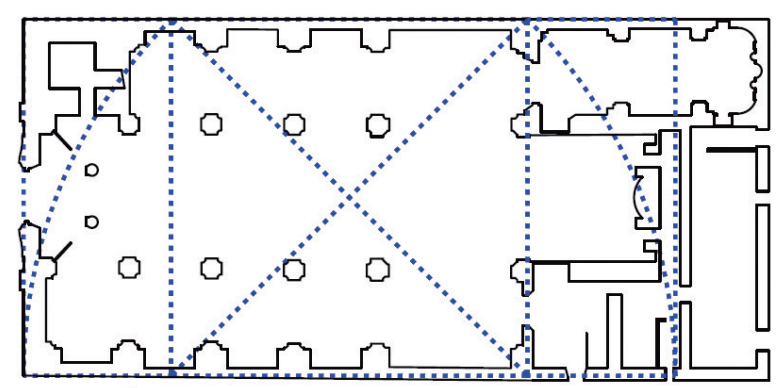

Proporción general del tempo

Figura 5. Modulación Iglesia de Suera. Fuente propia.

Olocau, plantea una posible relación con Joseph Dols, Joseph C. Ayora o algún colaborador directo de éstos (7, pp. 119, 122).

Del conjunto que compone el templo se deduce que fue construido de forma unitaria y puede inscribirse en un rectángulo de $43,15 \times 23,47 \mathrm{~m}$.

Es de reseñar la precisión en la ejecución del templo con una dispersión inferior al o,7\%.

Ribelles pretendió modificar la proporción del templo, estableciendo para todos sus elementos la proporción dupla, mientras que las trazas originales establecían una proporción 4/7 para la nave y de 7/13 en las capillas, que Ribelles aspiraba transformar en $8 / 16$ ( 5 , pp. 365, 515, 516). Sin embargo la métrica actual del templo responde a las proporciones de las trazas originales, con la proporción 4/7 (ver Figura 6).

Esta documentación, junto con la geometría real permite definir un módulo de medida $127 \mathrm{~cm}$ que equivale a 6 palmos de
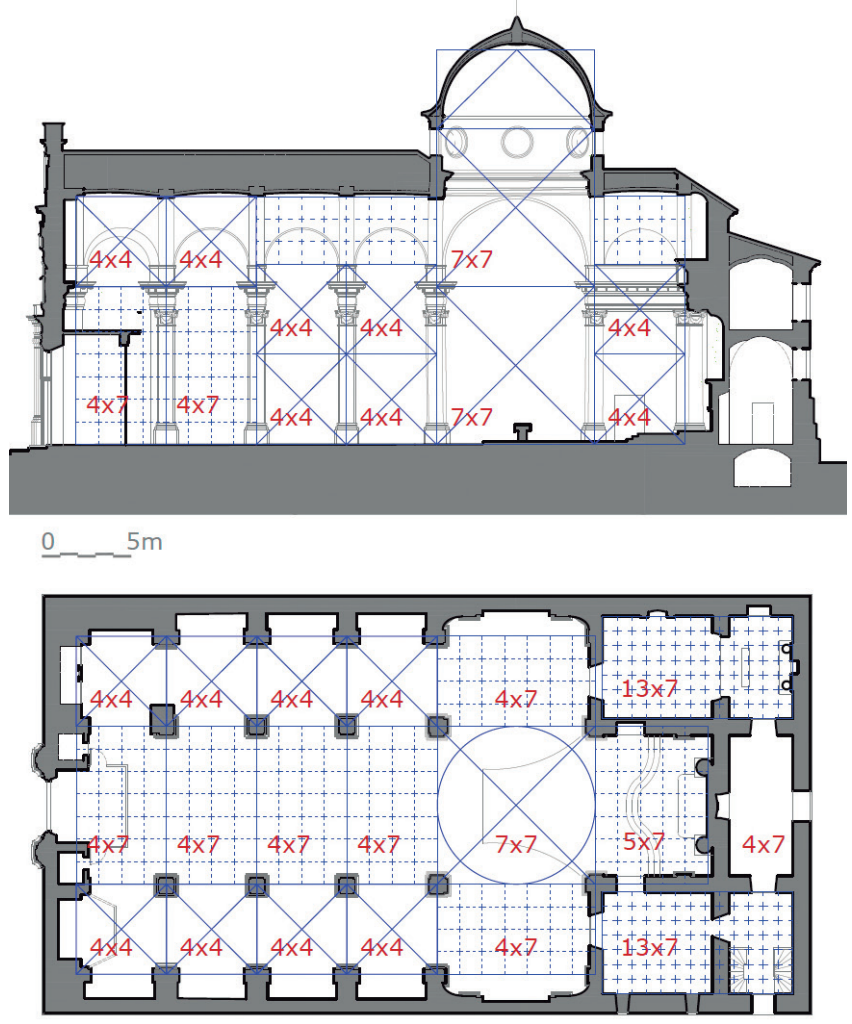

Modulación: 1vara $=109,9 \mathrm{~cm}$

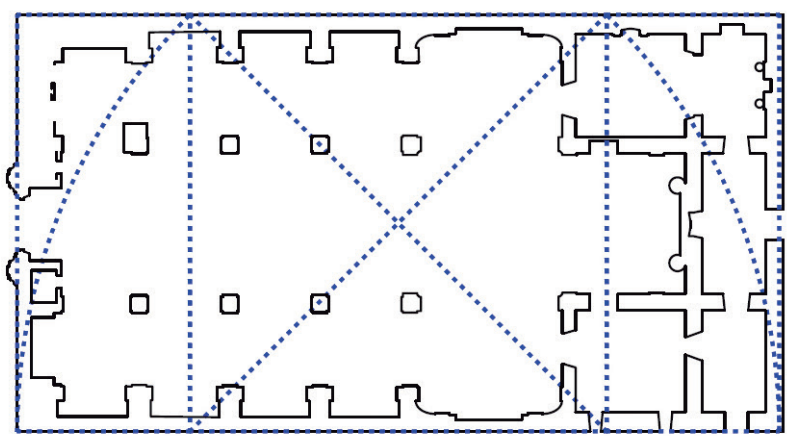

Proporción general del tempo

Figura 6. Modulación Iglesia de Les Coves de Vinromà. Fuente propia.

$21,1 \mathrm{~cm}$, con el que se definen los anchos de las naves principales, laterales y crucero.

\subsection{Iglesia de San Miguel de Quart de les Valls}

La construcción de este templo se supone que tuvo lugar en dos fases. En la primera se disponía de los planos desde finales de 1775 o principios de 1776 , comenzando las obras en febrero. En 1777, construidos los dos tramos de los pies, la torre y la fachada, se plantea el cambio de diseño, quedando paralizadas las obras. En la segunda fase se ejecutaría el resto del templo con un borrador del diseño solicitado en agosto de 1781, y aprobado 7 años más tarde, prolongándose la construcción desde 1789 hasta 1795. (7, pp. 152, 160)

Todo ello sitúa la ejecución de las obras del templo, como la antepenúltima delasiglesias de planta salón valencianas del xviII.

Cristóbal Bueno es el autor de la primera fase, y Vicente Cebrian de las trazas y la ejecución de la segunda fase, junto 
con el obrero Manuel Gomes y los maestros de obra Joseph y Vicente Cebrian, que comienzan a nombrarse a partir de 1777 .

Las dimensiones interiores de la nave incluyendo el tramo de los pies son de 16,24 x 38,34 m, suponiendo el presbiterio un incremento de su profundidad de 5,43 $\mathrm{m}$.

Del análisis de las medidas de la iglesia de Quart, (ver Figura 7), se desprende que puede existir una modulación de 10 varas en el ancho del crucero y naves centrales y 5 varas en el ancho de los tramos, cada una de ellas formada por 4 palmos de $21,8 \mathrm{~cm}$, ligeramente menor que el palmo valenciano (22,6 $\mathrm{cm})$. El trazado de los pilares es de 6 palmos, al igual que el espesor de las pilastras junto con el muro.

El estudio de los trazados geométricos pone de manifiesto que la planta responde a una proporción dupla en los tramos de la nave principal y del crucero. En el caso de las naves laterales la proporción utilizada es de 4/5 si se considera el centro de las pilastras, o de 5/5 si se tiene en cuenta la cara exterior del muro de fachada. En el caso de los tramos de los pies, se aprecia la utilización del Diagón en alguno de los espacios.
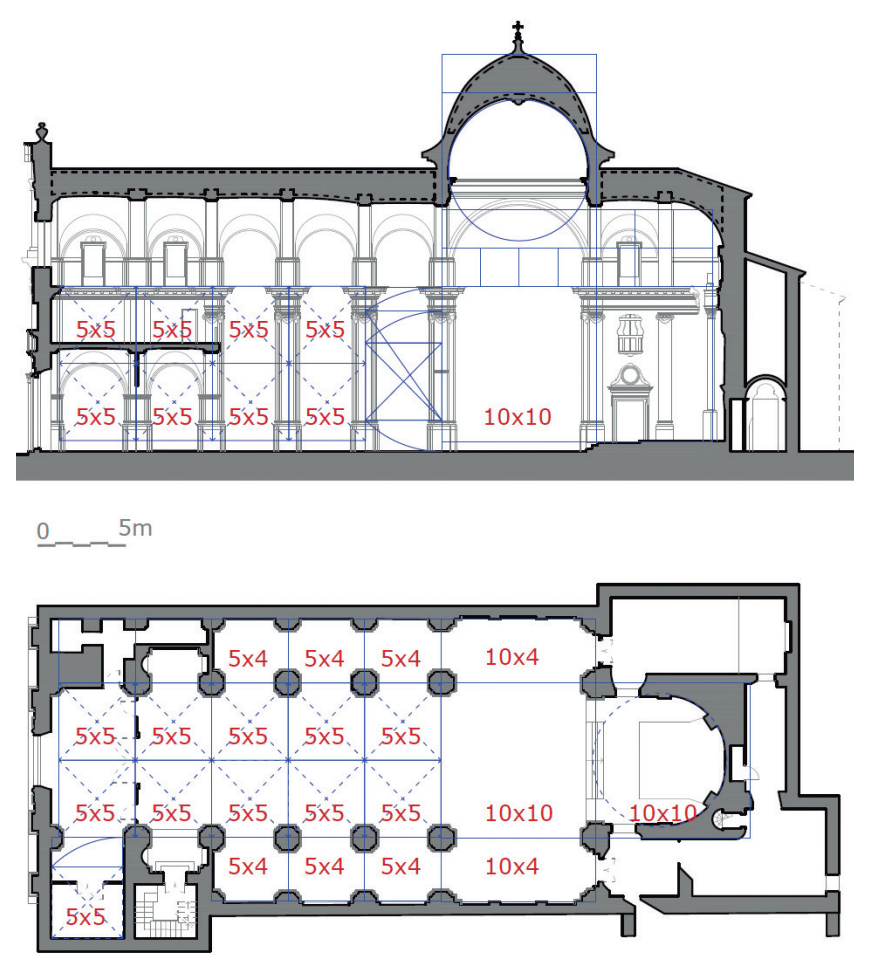

Modulación: 1vara $=87,4 \mathrm{~cm}$

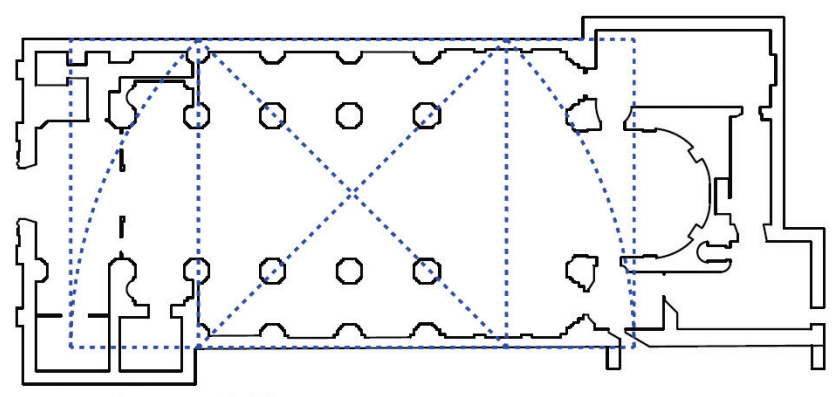

Proporción general del tempo

Figura 7. Modulación Iglesia de Quart de les Valls. Fuente propia.
En alzado no se ha podido determinar una modulacion concreta más allá de una aproximación ad quadratum con el ancho de la nave principal, y la posibilidad de encajar construcciones basadas en los trazados $\sqrt{ } 2$ y $\sqrt{ } 3$ para la definición de la proporción de la basa, fuste y capitel de las columnas.

En este caso, los diferentes recursos geométricos encontrados refuerzan la teoría de las diferentes fases constructivas, correspondiendo, cada una de ellas a los criterios propios de cada uno de los alarifes intervinientes.

Las irregularidades de la planta han podido cuantificarse en una dispersión del $0,7 \%$, mientras que en la sección longitudinal se aprecian diferencias de hasta $30 \mathrm{~cm}$ en la altura de las claves de los archos fajones, lo que supone una dispersión del $2,1 \%$.

\section{ANÁLISIS COMPARATIVO}

La diserción del análisis geométrico se estructura en cuatro niveles. Las proporciones generales exteriores, en las que se aprecian tres situaciones diferentes, la de Castell de Cabres, que se rige por la proporción próxima a 3/5, las de Cintorres, Suera y Les Coves de Vinromà que utilizan $\sqrt{ } 2 \mathrm{y}$ las de Vila-real y Benifairó de les Valls, en las que se aplica la sección Áurea, no quedando clara la proporción exterior del tempo de Quart de les Valls.

Sin embargo, respecto de estas proporciones exteriores, cabe precisar que las dimensiones reales difieren entre un $1,6 \%$ y un 2,4\% de las geométrias ideales, por lo que parece poco probable que se utilizarán durante el replanteo de estos. Del mismo modo, resulta complejo encajar la modulación de los espacios interiores en un rectángulo previo, por lo que se puede descartar esta hipótesis. Sin embargo si es probable que en la elección de la tipología del templo y la definición del número de tramos se considerara la adecuación del conjunto a alguna de las proporciones idealizadas.

La trama de los pilares y de los espacios interiores desvela que en la mayoría de las plantas se ha recurrido a modulaciones con proporciones geométricas del tipo 3/4, 3/5 y 4/7. Hecho que permite establecer un vínculo entre los templos de Castell de Cabres, Cinctorres, Benifairó de les Valls, Suera y Les Coves de Vinromà, y que desmarca a las de Quart de les Valls donde se ha utilizado la proporción Dupla, y a la de Vila-real que responde al Diagón.

La utilización de los recursos proporcionales dinámicos, tales como el rectángulo Áureo, el Diagón $\sqrt{ } 2$ y $\sqrt{ } 3$ han sido más difíciles de ajustar que las modulaciones basadas en entramados reticulares. De hecho, la proporción Áurea sólo se ha encontrado en la capilla de la Comunión y en la relación entre altura y separación de los tramos de Vila-real, y el Diagón en Vila-real y puntualmente en Quart de les Valls.

Tal como se aprecia en la tabla 3, en las iglesias de Suera y Les Coves de Vinromà se observa la utilización de trazados geométricos basados en la proporción 4/7 próximos a la construcción $\sqrt{ } 3$, mientras que en las de Cinctorres y Benifairó de les Valls se ha utilizado la proporción 3/5 cercana a la proporción Aurea, y en Castell de Cabres 3/4. 
Tabla 3. Trazados reguladores y Métrica

\begin{tabular}{|l|c|c|c|}
\hline \multirow{2}{*}{\multicolumn{1}{|c|}{ Iglesia }} & \multicolumn{3}{|c|}{ Trazados reguladores } \\
\cline { 2 - 4 } & Ext. & \multicolumn{2}{|c|}{ Interior } \\
\hline Castell de Cabres & $3 / 5$ & $3 / 4$ & $8 \times 8,6 \times 8,6 \times 6$ \\
\hline Cinctorres & $2 \sqrt{2-1}$ & $\begin{array}{c}3 / 5 \\
\equiv \text { Âurea }\end{array}$ & $\begin{array}{c}10 \times 10,6 \times 10,6 \times 6 \\
5 \times 5,3 \times 5,3 \times 3\end{array}$ \\
\hline Vila-real & Áurea & $\begin{array}{c}\sqrt{ } \mathbf{2} \\
\text { Aurea }\end{array}$ & $\begin{array}{c}14,5 \times 14,510 \times 14,5 \\
10 \times 10\end{array}$ \\
\hline $\begin{array}{l}\text { Benifairó de les } \\
\text { Valls }\end{array}$ & Áurea & $\begin{array}{c}3 / 5 \\
\equiv \text { Âurea }\end{array}$ & $\begin{array}{c}10 \times 10,6 \times 10,6 \times 6 \\
5 \times 5,3 \times 5,3 \times 3\end{array}$ \\
\hline Suera & $2 \sqrt{2}-1$ & $4 / 7 \equiv \sqrt{ } 3$ & $7 \times 7,4 \times 7,4 \times 4$ \\
\hline $\begin{array}{l}\text { Les Coves de } \\
\text { Vinromà }\end{array}$ & $2 \sqrt{2}-1$ & $\begin{array}{c}4 / 7 \equiv \sqrt{ } 3 \\
7 \times 7,4 \times 7,4 \times 4\end{array}$ \\
\hline $\begin{array}{l}\text { Quart de les } \\
\text { Valls }\end{array}$ & $\begin{array}{c}\text { Dupla, } \\
\sqrt{2^{1}} \\
\text { Áurea }\end{array}$ & $10 \times 10,5 \times 10,5 \times 4$ \\
\hline
\end{tabular}

\begin{tabular}{|c|c|c|c|c|}
\hline \multirow{3}{*}{$\begin{array}{l}\text { Iglesia } \\
\text { Castell de } \\
\text { Cabres }\end{array}$} & \multicolumn{4}{|c|}{ Métrica } \\
\hline & \multirow{2}{*}{\begin{tabular}{|l|} 
Unidad \\
Varas \\
\end{tabular}} & \multicolumn{2}{|c|}{ Equivalencia cm } & \multirow{2}{*}{$\begin{array}{c}\begin{array}{c}\text { Dispersión. } \\
\text { mediciones }\end{array} \\
<2,23 \% \\
\end{array}$} \\
\hline & & 79,4 & $=19,9 \times 4$ & \\
\hline Cinctorres & Brazas & 205,4 & $=22,8 \times 9$ & $<0,3 \%$ \\
\hline Vila-real & Varas & 90,9 & $=22,6 \times 4$ & $<0,3 \%$ \\
\hline $\begin{array}{l}\text { Benifairó de les } \\
\text { Valls }\end{array}$ & \begin{tabular}{|l|} 
Varas \\
Brazas \\
\end{tabular} & $\begin{array}{r}83,1 \\
166,2 \\
\end{array}$ & $\begin{array}{l}=20,8 \times 4 \\
=20,8 \times 8\end{array}$ & $<1,8 \%$ \\
\hline Suera & $\begin{array}{l}\text { Ancho } \\
\text { columna }\end{array}$ & 109,9 & $=21,99 \times 5$ & $<1,0 \%$ \\
\hline $\begin{array}{l}\text { Les Coves de } \\
\text { Vinromà }\end{array}$ & Cana & 127,0 & $=21,17 \times 6$ & $<0,7 \%$ \\
\hline $\begin{array}{l}\text { Quart de les } \\
\text { Valls }\end{array}$ & Varas & 87,0 & $=21,7 \times 4$ & $<0,7 \%$ \\
\hline
\end{tabular}

${ }^{1}$ Sólo se ha utilizado $\sqrt{ } 2$ para la configuración de los espacios en los dos tramos de los pies.

${ }^{2}$ La dispersión de mediciones se define como el porcentaje de la mayor diferencia de medida respecto de la media de todas las que deberían ser iguales, medidas a ejes.

El análisis de la métrica permite afirmar que, aunque no existe unanimidad en cuanto a las modulaciones detectadas, mayoritariamente éstas se aproximan a múltiplos del palmo del Reino de Valencia, salvo el caso de Castell de Cabres en el que el módulo está más próximo al palmo de Aragón o Tarragona.

Se ha puesto en evidencia la utilización del palmo valenciano en las iglesias de Vila-real y Suera, sin embargo, la realidad de la obra construida determina pequeñas variaciones de unidad de medida en ambos templos e incluso respecto del valor asignado por diversos autores (Tabla 1).

Por último, el análisis de la configuración morfólogica pone de manifiesto como primera conclusión que en todos los casos se trata de iglesias salón de planta longitudinal, a diferencia de lo que ocurre con el resto de las iglesias salón de la Comunidad Valenciana, en las que existen templos de planta centralizada (6).

En todos los casos se trata de iglesias de tres naves con un crucero, y tras el crucero el altar y otras dependencias. Sin embargo, Castell de Cabres y Benifairó tienen tres tramos en los pies, Quart cinco y las restantes cuatro.

Así mismo, se puede establecer una clara relación morfológica entre las iglesias de Les Coves, Suera y Vila-real. Respecto de la dos últimas Bautísta (7, p. 175) indica que la composi- ción es la misma, siendo las dimensiones generales de Suera la mitad. Sin embargo, la proporción de los espacios interiores en Les Coves y Suera se basan en el patrón 3/7, mientras que la de Vila-real $\sqrt{ } \mathbf{2}$.

\section{CONCLUSIONES}

La investigación y el análisis gráfico, realizados a partir del levantamiento de planos de las iglesias de planta salón valencianas relacionadas con el arquitecto Ayora y su saga, permiten establecer con contundencia una serie de conclusiones respecto a los recursos geométricos de sus trazados, las unidades de medidas empleadas y la vinculación de éstas con el maestro.

El estudio realizado pone de manifiesto la aplicación de forma mayoritaria de trazados basados en proporciones racionales o estáticas tales como $3 / 4$ o $3 / 5$ recogidas en los tratados clásicos y otras como $4 / 7$ (próxima a $\sqrt{3}$ ) tan sólo cabe plantear la hipótesis de la utilización de proporciones irracionales o dinámicas como el Diagón o la proporción Áurea en la de Vila-real y puntualmente en Quart de les Valls.

En relación con la autoría de cada uno de los templos, aunque resulta complejo afirmar o corroborar autorías en base a sus trazados reguladores, puesto que en general se han utilizado proporciones de uso corriente en la arquitectura clásica, sí es viable enunciar una serie de afirmaciones.

Por un lado, es posible establecer un vínculo común entre Castell de Cabres, Cinctorres, Suera, Benifairó de les Valls y Les Coves de Vinromà, templos que basan sus trazas de planta en la combinación de proporciones estáticas que seguramente buscan la belleza espacial desde la sencillez del trazado, por lo que, si en el diseño no intervino Joseph Cristóbal Ayora o sus hermanos, lo hicieron otros maestros constructores muy próximos, y seguramente con los mismos conocimientos y criterios.

De otro modo la planta de Quart de les Valls donde se recurre a la proporción Dupla, mucho más sencilla, permite descartar la intervención del maestro Ayora en la misma.

Y el particular trazado de la arciprestal de Vila-real, que, respondiendo únicamente a la aplicación de trazados clásicos, se desmarca del resto, lo que permite afirmar que, en la continuación de las obras, tras la muerte de Nadal, Ayora se limitó a seguir fielmente los planos definidos por aquel.

En cuanto a las unidades de medida empleadas, el estudio realizado confirma la convivencia de diferentes sistemas métricos. A pesar de la documentación de unas unidades de medida delimitadas por provincias, lo cierto, es que no se disponía de un sistema métrico único, sino que en cada caso utilizaban un patrón de medida, bien sea el propio de la población del templo o de la originaria de los alarifes o constructores.

Habitualmente se aproximaba al palmo valenciano o a sus múltiplos de vara, braza o cana, existiendo generalmente una gran correspondencia entre el templo construido y el modelo idealizado con la hipótesis de modulación. En este sentido se pueden vincular todos los templos a través de la utilización de unidades de medida valencianas, donde quedaría al margen la iglesia de Castell de Cabres. Sin embargo, dado que la autoría 
por parte de Ayora es un hecho confirmado en este templo, y siendo ésta una sus primeras iglesias salón construidas en tierras valencianas, esta situación viene a confirmar la adaptación del maestro a las condiciones técnicas del nuevo entorno.
Por otro lado, en el templo de Vila-real, la utilización de nuevo de la métrica aragonesa en la configuración de las portadas laterales, respaldan las supuestas intervenciones puntuales de Ayora y de paisanos suyos.

\section{REFERENCIAS}

(1) Muñoz Jiménez, J.M. (1996). Las iglesias de salón en la provincia de Guadalajara. Wad-al-Hayara: revista de estudios de Guadalajara, 23: 274-275.

(2) Martínez Frías, J.M. (1980). El gótico en Soria. Arquitectura y escultura monumental, pp. 358-371, Salamanca: Diputación provincial.

(3) Bérchez Gómez, J. (1997). El arte de la Edad Moderna Iberoamericana. En Ramírez, J.A. (coord.) Historia del Arte: La Edad Moderna (pp. 381). Madrid: Alianza editorial.

(4) Pano Gracia, J.L. (2004). El modelo de planta salón: origen difusión e implantación en América. En Lacarra Ducay, M.C. (coord.) Arquitectura religiosa del siglo XVI en España y Ultramar (pp. 39-84). Zaragoza: Diputación.

(5) Gil Saura, Y. (2004). Arquitectura Barroca en Castellón. Castellón: Diputación.

(6) Sáez Riquelme, B. (2017). Características formales de las iglesias salón valencianas del s. XVIII. EGA. Revista de expresión gráfica arquitectónica, 22(29): 38-47. https://doi.org/10.4995/ega.2017.1463

(7) Bautista i García, J.D. (2002). Esglésies-saló del segle XVIII a les comarques valencianes, pp. 47, Castellón: Fundación Dávalos-Fletcher.

(8) Martín Talaverano, R. (2014). Documentación gráfica de edificios históricos: principios, aplicaciones y perspectivas. Arqueología de la Arquitectura, 11: e011. https://doi.org/10.3989/arq.arqt.2014.014

(9) Sáez Riquelme, B. y Pitarch Roig, A.M. (2016). Combinación de tecnologías en el levantamiento de planos. La iglesia parroquial de Portell. EGE: revista de expresión gráfica en la edificación, 9: 12-19. https://doi.org/10.4995/ ege.2016.12449

(10) García Ortega, A.J. (2012). ¿Cómo se traza una iglesia gótica? Algunas claves y un caso concreto. EGA: expresión gráfica arquitectónica, 7: 194-201. https://doi.org/10.4995/ega.2012.1441

(11) Moreno Alcalde, M. (1994). La iglesia de Santa Clara de Briviesca (Burgos). Hipótesis sobre el trazado de su planta. Anales de Historia del Arte, 4: 191-201.

(12) López González, M.C. y García Valldecabres, J. (2012). Una metodología para el análisis de los trazados reguladores clásicos de la arquitectura. EGE: revista de expresión gráfica en la edificación, 7: 108-144. https://doi.org/10.4995/ ege.2012.12506

(13) Roldán Medina, F.J. (2015). La doble medida en la arquitectura del pasado: El Palacio de Onda y su planta antropométrica. Arqueología de la Arquitectura, 12: e023. https://doi.org/10.3989/arq.arqt.2015.005

(14) Torner, Ma .E., Más, Á., Lerma, C., Gil, E. (2015). Metodología para el estudio de las trazas y las relaciones intrínsecas en la Iglesia de la Asunción de Llíria. Informes de la Construcción, 67(538): eo70, doi: https://doi.org/10.3989/ic.13.118

(15) Roldán Medina, F.J. (2012). Combinación de módulos en el sistema metrológico clásico. EGE: revista de expresión gráfica en la edificación, 7: 26-33. https://doi.org/10.4995/ege.2012.12498

(16) Roldán Medina, F.J. (2013). Combining classical metrology models. Virtual Archaeology Review, 4(9): 82-92. https:// doi.org/10.4995/var.2013.4252

(17) Gentil Baldrich, J.M. (2008). Sobre la Proporción y los Trazados Geométricos de la Arquitectura. En Soler Sanz, F. Trazados reguladores octogonales en la arquitectura clásica, (pp. 15-24). Valencia: General de Ediciones de Arquitectura.

(18) Serlio Boloñes, S. (1545). El primo libro d'architectrura, (20v-21r), Paris: J. Barbé. Recuperado de https://archive.org/ details/HArteRo7T19 [consultado el 13/05/2017]

(19) Arfe y Villafañe, J. (1585). De varia commensuración para la Esculptura y Architectura. (Cap VII). Sevilla: Albatros Ediciones.

(20) Pérez Galdeano, A.M. (2009). Algunas consideraciones sobre la difusión de los tratados de arquitectura en Hispanoamérica (siglos XvI-XVII). Cuadernos de arte de la Universidad de Granada, 40: 107-118. Recuperado de http://revistaseug.ugr.es/ index.php/caug/article/download/257/249

(21) Chalmeta Gendrón, P. (2008). El almotacén a través de los llibre del Mustaçaf. Aragón en la Edad Media, 20: $203-223$.

(22) València (Regne). Corts. (1547): Fori Regni Valentiae. Arte ac Industria experti viri loannis de Mey Flandri. Valencia.

(23) Real Orden de 9 de diciembre de 1852, por la que se determinan las tablas de correspondencia recíproca entre las pesas y medidas métricas y las actualmente en uso (Diccionario jurídico-administrativo. Madrid, 1858) (Centro Español de Metrología).

(24) Soler Sanz, F. (2008). Trazados reguladores octogonales en la arquitectura clásica, pp.15-24. Valencia: General de Ediciones de Arquitectura.

(25) Sáez Riquelme, B. (2013). Iglesias salón valencianas del XVIII: levantamiento gráfico, análisis gráfico y constructivo, patología común (Tesis doctoral). Castellón: Universitat Jaume I. Recuperado de http://hdl.handle.net/10803/298717

(26) Sáez Riquelme, B. y Pitarch Roig, A.M. (2012). Algunas lesiones comunes de las iglesias salón: San Pedro en Cinctorres, San Jaime en Vila-real y San Martín en Callosa de Segura. En IV Congreso de patología y rehabilitación de edificios. Galicia: PATORREB.

(27) Nadal, J.J. (1753). Planos Originales de la iglesia arciprestral de San Jaime de Villarreal (Castellón): planta, sección transversal y dos longitudinales. Archivo Histórico Nacional. Consejos, (MyP 2655-2658). Madrid.

(28) Traver García, B. (1909). Historia de Villarreal. Villarreal: Establecimiento tipográfico de Juan Botella. 
(29) Sáez Riquelme, B. y Pitarch Roig, A.M. (2010). Geometry in Hall Churches: Callosa de Segura, Portell, Cinctorres and Vila-real. En APEGA 2010. X Graphic Expression Applied to Building International Conference, (Vol. II, pp.150-167). Ed. Marfil, Universidad de Alicante.

(30) Navarro Esteve, P. y Herráez Boquera, J. (2007). Levantamiento de planos. En Campos González, C. [et al.], Proyecto de Restauración integral de la iglesia de Sant Jaime de Vila-real. Valencia: Conselleria de Cultura i Esport, Gerencia de la Fundación "La Llum de les Imatges".

(31) Gimeno i Estornell, V. (2006). Notícies per a la història de la Vila i parròquia de Suera, pp.152-155, Castellón: Diputació. 